\title{
Inversion and gain in hydrogenic ion levels induced by photoionization pumping
}

\author{
D. G. Goodwin and E. E. Fill \\ Max Planck Institut für Quantenoptik, D-8046 Garching, Federal Republic of Germany
}

(Received 14 December 1987; accepted for publication 24 March 1988)

\begin{abstract}
If a low- $Z$ plasma is subjected to ionizing radiation, stationary inversions on hydrogenic ion level populations occur. The plasma conditions and pumping requirements to achieve gain on the Lyman- $\alpha$ and Balmer- $\alpha$ lines are investigated by means of a collisional-radiative model. The calculations are carried out for two cases: first, for the case in which the electron temperature is determined solely by radiative cooling, and second, for the case in which additional electron cooling is provided. In the first case, measurable gain on the Balmer- $\alpha$ line is found, athough the Lyman- $\alpha$ transition remains uninverted. In the second case, the Balmer$\alpha$ gain is dramatically enhanced, and sizable Lyman $\alpha$ gain is predicted.
\end{abstract}

\section{INTRODUCTON}

The challenge to develop new lasers at ever shorter wavelengths has led to several proposals and experiments at soft $x$-ray wavelengths, most of which have involved the genexation of highly ionized species in a laser plasma. Notable successes have been achieved with the electron collisional pumping of $\mathrm{Ne}-1 \mathrm{lik} \mathrm{e}^{1,2}$ or $\mathrm{N}^{3}-\mathrm{like} \mathrm{e}^{3}$ ions, with gain on $3 s-3 p$ or $4 p-4 d$ transitions, respectively, and with recombination pumping of hydrogenic ions, which led to the observation of gain on the Baimer- $\alpha$ transition. ${ }^{4}$

Various schemes to optically pump an $\mathrm{x}$-ray laser have been proposed, most of which have involved matched-line pumping. ${ }^{5,7}$ However, none of these have as of yet been successfully demonstrated. In this article, we present a study of an optical pumping scheme which has only occasionally appeared in the literature, ${ }^{8-10}$ namely, pumping with ionizing radiation.

This excitation mechanism may be thought of as the $x$ ray analog of fashlamp pumping of, for example, a ruby or Neodymium laser where the pumping radiation (of relative ly broad spectral composition) excites a continuum of levels, from which relaxation into the upper laser level occurs. Boiko et al. ${ }^{9}$ noted using relatively simple analytic estimates that inversions between hydrogenic levels $n=4$ and $n=3$ should occur if ionizing radiation is applied to a low- $Z$ plasma. We show in this article that it is also possible to achieve inversions between $n=3$ and $n=2$ (Bamer- $\alpha$ ), and even between $n=2$ and $n=1$ (Lyman- $\alpha$ ).

Such a scheme could be implemented by using the $x-r a y$ emission from a relatively high- $Z$ laser plasma to pump a low-Z target plasma. Various configurations are conceptually possible: The two plasmas could be in contact with one another, which naximizes the geometric coupling but makes difficult the separate control of the density and ternperature of the two plasmas. Aiternatively, the two plasmas could be physically separate, with the $x$-ray emission from the pump plasma coupled to the target plasma with focusing $x$-ray multilayer mirrors. The target plasma could be created with a prepulse from the laser, allowing it to expand to the desired density and temperature before the $x$-ray pumping pulse arrives, or could be created directly by the $x$-ray puise, since an intense $x$-ray pulse can fully strip a low- $Z$ atom in a few hundred picoseconds or less. This second possibility is attractive for situations in which a high density is desired, since there is no limitation due to the laser critical density, as there is in a laser-created plasma.

This pumping scheme may have certain advantages in comparison to recombination pumping of hydrogenic ions. In the recombination scheme, very short laser pulses are required, and the conditions needed to achieve adequate hydrodynamic cooling can be quite severe, particularly as $Z$ is increased. For example, Pert has calculated that optimum gain conditions for a recombining aluminum plasma require an initial density greater than solid density. ${ }^{11}$ The present scheme is not subject to these constraints, and in addition has the possibility of achieving Lyman- $\alpha$ inversions, as well as Balmer $\alpha$ inversions. On the other hand, an intense source of $x$ rays is required, which can decrease the overall effciency of the scheme, since losses are associated with the conversion of laser light to $x$ rays and with the coupling of the $x$ rays to the target.

In this work, we use a detailed collisional-radiative model to investigate the plasma conditions and pump intensities needed to obtain inversion on the Balmer- $\alpha(3 \rightarrow 2)$ and Lyman- $\alpha(2 \rightarrow 1)$ transitions, and the magnitude of the attainable gain coeficients. To concentrate on the underlying plyysics, we consider only the simplest possible case: a steady-state uniform plasma subjected to ionizing radiation. That this idealized situation is relevant to an actual experiment follows from consideration of the time scales involved: the relaxation times of the excited level populations $\left(\approx 10^{-12} \$\right)$ are always much shorter than the time scale for hydrodynamic expansion $\left(\approx 10^{-9} \mathrm{~s}\right.$ ), and thus the excited state populations evolve in a quasistatic manner. Furthermore, the ground state also evolves quasistatically for suffciently strong pumping, since its relaxation time is determined primarily by the photoionization rate.

Transient effects do come into play when the plasma temperature is considered. At steady state, the temperature is determined by the balance between the power absorbed from the pump and that radiated away into full solid angle. However, during the short duration of the pump pulse, this "power balance" temperature may not be attained. Further- 
more, additional cooling due to hydrodynamic expansion or conduction may result in a temperature lower than that calculated for radiative cooling alone (see Appendix). Consequently, we consider both the case in which the temperature is given by power balance (i.e., radiative cooling alone) and that in which the temperature is below the power balance value.

We confine our attention to the case of monochromatic pump radiation with a photon energy greater than the ionization potential $\left(h v_{p}>13.6 Z^{2} \mathrm{eV}\right)$. The opposite extreme of blackbody radiation has been shown previously to be less favorable for producing inversions. ${ }^{10}$ Furthermore, the spectrum of a laser plasma is far from blackbody, and usually consists of strong line emission. The assumption of monoch romaticity conld be easily relaxed without changing any essential physics by integrating over the pump radiation spectrum (as long as bound-bound transitions are not pumped).

\section{THEORY}

\section{A. Pumping rates}

The ionizing radiation removes electrons from the various hydrogenic levels into the continuum. The ionization rate from level $n$ may be written as

$$
-\left(\frac{d B_{n}}{d t}\right)_{\text {purrs }}=P_{n} B_{n},
$$

where $B_{n}$ is the population density in level $n$ (assuming the $(l, j)$ subleveis are populated statistically). The ionization rate per ion $P_{n}$ is given by

$$
P_{n}=\sigma_{n} n_{\mathrm{ph}},
$$

where $\sigma_{n}$ is the cross section for photoionization from level $n$, and $n_{\mathrm{ph}}$ is the photon flux per $\mathrm{cm}^{2}$ per second. The photoionization cross-section $\sigma_{n}$ can be expressed by the Kramers formula as ${ }^{12}$

$$
\sigma_{n}=1.98 \times 10^{-14} Z^{4} G_{n f} /\left[\left(h v_{p}\right)^{3} n^{5}\right] \quad \mathrm{cm}^{2},
$$

where $G_{n f}$ is the bound-free Gaunt factor of level $n$ and $h v_{p}$ is the energy of the pump photons in $\mathrm{eV}$. From Eqs. (2) and (3), the photoionization rate for level $n$ is related to that for the ground level by

$$
P_{n}=\left(P_{1} / n^{5}\right)\left(G_{n f} / G_{1 j}\right)
$$

Equation ( 1 ) is not valid at very high pump rates, as the stimulated emission from the continuum leads to a saturation which must be taken into account. The complete expression including stimulated emission is ${ }^{13}$

$$
-\left(\frac{d B_{n}}{d t}\right)_{\text {pump }}=P_{n}\left[B_{n}-B_{n}^{*} \exp \left(-\frac{h v_{p}}{k T_{e}}\right)\right]
$$

where $B_{n}^{*}$ is the LTE value of the number density of ions in level $n$, given by

$$
\begin{aligned}
B_{n}^{*}= & 1.66 \times 10^{-22} g_{n} N_{e} N_{1}\left(k T_{e}\right)^{-3 / 2} \\
& \times \exp \left(E_{n} / k T_{e}\right) \mathrm{cm}^{-3} .
\end{aligned}
$$

Here $g_{n}$ is the level degeneracy $\left(2 n^{2}\right), N_{e}$ is the electron density $\left(\mathrm{cm}^{-3}\right), N_{1}$ is the density of bare nuclei $\left(\mathrm{cm}^{-3}\right)$, $k T_{e}$ is the electron temperature (eV), and $E_{n}$ is the ionization potential of level $n$, equal to $13.6 \mathrm{Z}^{2} / n^{2} \mathrm{eV}$.
The pumping rates, e.g., $P_{1}$, may be easily expressed in terms of the radiation intensity. From Eqs. (2) and (3) and using $I_{p}=h v_{p} n_{\mathrm{ph}}$, one has for the intensity

$$
I_{p}=8.2 \times 10^{-6} P_{I}\left(h v_{p}\right)^{4} Z^{-4} G_{1 f}^{-1} \quad \mathrm{~W} / \mathrm{cm}^{2} \text {, }
$$

where $h v_{p}$ is in $\mathrm{eV}$. It should be noted that the pump intensity needed to produce a giver pumping rate $P_{1}$ is proportional to $Z^{4}$, since the required energy of the pumping photons $\left(>13.6 Z^{2}\right)$ scales as $Z^{2}$.

\section{Power balance}

If the plasma is treated as an isolated system and hydrodynamic cooling is neglected, then in steady state the power absorbed from the pump must equal the power radiated into the full solid angle. This condition determines the steadystate temperature reached by the plasma.

Assuming the only ions present in the plasma to be bare nuclei with charge $Z$ and hydrogenic ions with charge $Z-1$, the pump power absorbed by the bound-free transitions is given by

$$
A_{b f}=h v_{p} \sum_{n} P_{n}\left[B_{n}-B_{n}^{*} \exp \left(-\frac{h v_{p}}{k T_{e}}\right)\right]
$$

The power absorbed in free-free transitions can be derived from the free-free absorption coefficient of Spitzer ${ }^{12}$ :

$\kappa_{v}=3.69 \times 10^{8} \frac{\overline{z^{2}} N_{e} N_{i}}{T_{e}^{1 / 2} v^{3}}\left[1-\exp \left(-\frac{h v}{k T_{e}}\right)\right] G_{f f} \mathrm{~cm}^{-1}$.

Here $N_{i}$ is the total ion density, $T_{e}$ is the electron temperature in $\mathrm{K}, G_{f f}$ is the free-free Gaunt factor, and $z^{2}$ is the average value of the square of the ion charge, which, if most of the ions are fully stripped, is nearly equal to $Z^{2}$. The exponential in Eq. (9) accounts for stimulated emission from the electron gas with a Maxwellian velocity distribution. The absorbed power is given by $A_{f f}=n_{\mathrm{ph}} h v_{p} \kappa_{v}$, which together with Eqs. (2), (3), and (9) results in the expression

$$
\begin{aligned}
A_{f f}= & 1.2 \times 10^{-23} P_{1}\left(\frac{h v_{p}}{Z^{2}}\right)\left(\frac{z^{2}}{Z^{2}}\right) \frac{N_{e} N_{i}}{\sqrt{k T_{e}}} \\
& \times\left[1-\exp \left(-h v_{p} / k T_{e}\right)\right] G_{f j} \mathrm{eV} / \mathrm{cm}^{3} \mathrm{~s},
\end{aligned}
$$

where $k T_{e}$ is in eV.

The power radiated by the plasma consists of three contributions: bound-bound, free-bound, and free-free radiation. For bound-bound radiation, the enitted power is obtained by summing over all spontaneous decay channels

$$
P_{b b}=\sum_{n} \sum_{n^{\prime}<n} B_{n} A_{n n^{\prime}}\left(E_{n}-E_{n^{\prime}}\right),
$$

where $A_{n n^{\prime}}$ is the Einstein $A$ coefficient for the transition $n \rightarrow n^{\prime}\left(n>n^{\prime}\right)$.

The power radiated in free-bound transitions can be obtained by summing over all final levels as

$$
P_{f b}=N_{e} N_{1} \sum_{n} \int_{0}^{\infty} h v \sigma_{n}(v) n_{e}(v) v d v
$$

where $n_{e}(v)$ is the normalized velocity distribution of the electrons, and $h v=m_{\mathrm{e}} v^{2} / 2+E_{n}$. The quantity $\sigma_{n}(v)$ is the cross section for radiative recombination ${ }^{12}$ 


$$
\sigma_{n}(v)=2.11 \times 10^{-22} \frac{\left(E_{n}\right)^{2}}{h v\left(m_{e} v^{2} / 2\right)} \frac{G_{f n}}{n^{3}}
$$

with all units in cgs. Here $G_{f n}$ is the free-bound Gaunt factor. Assuming a Maxwellian velocity distribution for the electrons, the integration in Eq. (12) can be carried out and one obtains

$P_{f b}=2.61 \times 10^{-12} Z^{4} \frac{N_{e} N_{1}}{\sqrt{k T_{e}}} \sum_{n} \frac{G_{f n}}{n^{3}} \mathrm{eV} / \mathrm{cm}^{3} \mathrm{~s}$.

The free-free emission is given by ${ }^{12}$

$P_{f j}=9.56 \times 10^{-14} \bar{z}^{2}\left(N_{e} N_{i} / \sqrt{k T_{e}}\right) G_{f j} \mathrm{eV} / \mathrm{cm}^{3} \mathrm{~s}$.

The power balance condition then reads

$$
P_{b b}+P_{f b}+P_{f f}=A_{b f}+A_{f f} \text {. }
$$

Since each of these quantities is a function of temperature, either explicitly or implicitly through the level populations, the power balance temperature $T_{\mathrm{pb}}$ may be determined as that temperature which satisfies Eq. (16).

\section{Fine-structure effects}

The fine-structure splitting of the hydrogenic energy leveis has two consequences which need to be considered. First, the splitting can be large enough that the electroncollisional transition rates between the various $(l, j)$ sublev. els with the same $n$ are insuficient to maintain statistical equilibrium and thus their relative populations are no longer proportional to their degeneracies. In such cases, separate rate equations must be solved for each sublevel, rather than just for each energy level. Furthermore, the fine-structure splitting of the energy levels splits each spectral line into several components, which must be taken into account in computing the gain coefficient. The Lyman- $\alpha$ line is split into two and the Balmer- $\alpha$ line into seven fine-structure components. Under high-density, high-temperature conditions, however, only the structure relevant to level $n=2$ is resolved, resulting in an apparent doublet for both lines (although for very low $Z$, line broadening obscures the doublet structure).

Fortunately, it is only necessary to write separate rate equations for the sublevels of the first few energy levels, as the fine-structure splitting decreases with $n$ as $n^{-3}$. Sampson ${ }^{14}$ has given a criterion for statistical equilibration among sublevels which indicates that for the range of electron densities and temperatures of interest here, equilibration may be assumed for $n \geqslant 4$.

\section{Line broadening}

Since the gain depends sensitively on the linewidth, it is necessary to estimate the various broadening mechanisms with reasonable accuracy. Both Doppler and Stark broadening contribute to the width. If the ion temperature is taken to be equal to the electron temperature, then the thermal Doppler broadening is given by

$$
\Delta v_{D} / v=5.43 \times 10^{-5}\left(k T_{e} / Z\right)^{1 / 2},
$$

assuming the ion atomic weight $W=2 \mathcal{Z}$.

The dominant Stark broadening contribution is the quasistatic ion broadening (Holtsmark broadening), the linewidth of which may be esimated as ${ }^{15,16}$

$$
\Delta v_{H}=2\left(n^{2}-n^{\prime 2}\right) N_{i}^{2 / 3} s^{-1} \text {. }
$$

This mechanism is only effective, however, for line components which exhibit a linear Stark shift. The Baimer- $a$ and Lyman $a$ lines both contain unshifted components $(2 / 3$ of the total line strength for Lyman- $\alpha$, and $1 / 3$ of the line strength of the dominant $3 D_{5 / 2}-2 P_{3 / 2}$ component of the Balmer- $\alpha$ line), which are unaffected by Holtsmark broadening, and are broadened only by Doppler and dynamic Stark broadening.

Both the electrons and the ions contribute to the dynamic Stark broadening. The electron broadening may be estimated using the formula given by Sobelman et al, ${ }^{15}$ which may be written in mixed units as

$$
\begin{aligned}
\Delta v_{e}= & 6.8 \times 10^{-8} N_{e}\left(k T_{e}\right)^{-1 / 2} \\
& \times\left[\ln \left(k T_{e} / \sqrt{N_{e} I}\right)+24.90\right] I \mathrm{~s}^{-1},
\end{aligned}
$$

where $I=27 / Z^{2}$ for almer- $\alpha$, and $17 /\left(2 Z^{2}\right)$ for Lyman- $\alpha$.

There is, at present, no simple way to compute the broadening due to ion dynamic effects short of numerical simulation. However, Stamm et al. ${ }^{17}$ have shown that the primary effect of ion dynamics is to cut of the dipole correlation function for times long compared to the inverse ion plasma frequency $\omega_{p i}^{-1}$. Consequently, we have adopted the simple expedient of adding a contribution to the electron broadening equal to the ion plasma frequency to allow approximately for additional broadening due to ion dynamics. Thus, the full-width broadening due to ion dynamical effects may be estimated as

$$
\Delta v_{i}=297 \sqrt{2 N_{i}} \quad \mathrm{~s}^{-1} \text {. }
$$

The central component width computed in this manner $\left(\Delta v_{e}+\Delta v_{i}\right)$ agrees well with the width given by Oza, Greene, and Kelleher ${ }^{38}$ for the Bamer $\alpha$ line of $C^{5+}$ at $T_{e}$ $=20 \mathrm{eV}$ ( $a$ typical temperature for $\mathrm{X}$-ray laser experiments with hydrogenic carbon).

Putting typical parameter values into Eqs. (17)-(20), one finds that the Holtsmark broadening is generally far larger than the other widths, which leads to the conclusion that the Holtsmark-broadened components make little contribution to the gain. For example, if we take $N_{e}=5 \times 10^{20}$ $\mathrm{cm}^{-3}, T_{e}=100 \mathrm{eV}$, and $Z=9$, then $\Delta v_{D}=6 \times 10^{12} \mathrm{~s}^{-1}$, $\Delta v_{H}=1.4 \times 10^{14} \mathrm{~s}^{-1}, \Delta v_{e}=7.6 \times 10^{12} \mathrm{~s}^{-1}$, and $\Delta v_{i}$ $=6.6 \times 10^{12} \mathrm{~s}^{-1}$. The Doppler, electron impact, and ion $d y-$ namic widths are seen to be all of the same order of magnitude, and thus one may not, in general, assume that Doppler broadening is dominant in hydrogenic ions for conditions typical of $x$-ray laser experiments.

Taking all broadening mechanisms into account, the Pine shape can be approximately described as the sum of two pronles: a central "unshifted" component, broadened only by Doppler and dynamic Stark broadening, superimposed on a much broader profile, resuiting from the Holtsmarkbroadened components. The fractional area under the central component is $2 / 3$ for Lyman- $\alpha$, and $1 / 3$ for Bamer $\alpha$ $3 D_{5 / 2}-2 P_{3 / 2}$. 


\section{NUMERLGA RODEL}

A collisional-radiative code including the photoionization terms discussed above was used to calculate the steadystate populations of the various hydrogenic levels. The code allows for the presence of three ionization stages: bare nuclei, hydrogenic ions, and heliumbe ions, and considers the effects of the ine-structure splitting of the hydrogenic ion leveis. The system of rate equations for the populations of each level for the hydrogenic and heliumlike ions is solved in the steady-state limit, accounting for all relevant collisional and radiative processes; namely (a) electron-collisional excitation, de-excitation, and ionization, (b) radiative decay, (c) radiative, three-body, and dielectronic recombination, and (d) photoionization due to the pump radiation, allowing also for stimulated recombination [Eq. (5)]. The plasma is assumed to be optically thin for all transitions.

The temperature may be either specified, or, for the power balance case, computed by solving the systern of rate equations iteratively until the temperature is found which satisfies Eq. (16).

It should be noted that the expressions given to compute the pumping rates and power balance were written assuming the only ions present in the plasma are the hydrogenic and the completely stripped ions, and that the hydrogenic levels could be characterized by $n$ alone. In the calculations, however, more complete versions of these expressions are employed, accounting where necessary for the $(l, j)$ subleveis of the hydrogenic ions and for the presence of heliumlike ions.

The hydrogenic ion energy leveis through $n=7$ are included in the model, with higher levels being lumped into the continumm. The sublevels are considered individually for $n \leqslant 3$, while for $4 \leqslant n \leqslant 7$ they are assumed to be populated statisticaliy, so that only rate equations for the level populations as a whole need be solved.

The heliumlike ions are handled in an approximate manner, since in the present work they are of interest only as a sink term for the hyarogenic population. The He-like ions are treated in the high- $Z$ (hydrogenic) limit, with an effective $Z$ accounting for shielding. Levels up to $n=7$ are considered, with the $(l, j)$ spliting neglected. Doubly excited states are likewise neglected.

The collisional and radiative rate coefficients are taken from the literature, and are derived assuming a Maxwellian electron velocity distribution. The collisional excitation coefficients of Golden et al. ${ }^{20}$ and Clark ef al. are used for transitions $(n l j) \rightarrow\left(n^{\prime} l^{\prime} j^{\prime}\right)$, where $n, n^{\prime} \leqslant 5$, and $n<n^{\prime}$. The $\left(j, j^{\prime}\right)$ dependence of the coeficients occurs only through an angular momentum couphing factor, given by ${ }^{15}$

$Q_{k}\left(j_{j} l^{\prime} j^{\prime}\right)=(2 l+1)\left(2 j^{\prime}+1\right)\left[\begin{array}{ccc}l & j & 1 / 2 \\ j^{\prime} & l & \kappa\end{array}\right\}^{2}$

Here $k$ is the multipole order for the transition, which is taken to be the lowest value for which $Q_{\kappa}$ is nonzero, i.e., $\kappa=|l-i|$. Where necessary, the coefficients are summed over $\left(l^{\prime}, j^{\prime}\right)$ and/or averaged over $(l, j)$. When summed over $j^{\prime}, Q_{k}$ reduces to 1.0 , and when averaged over $j, Q_{k}$ reduces to the relative statistical weight of the final state $\left(2 j^{\prime}+1\right) /\left[2\left(2 l^{\prime}+1\right)\right]$. For transitions $(n) \rightarrow\left(n^{\prime}\right)$ and transitions $(n l j) \rightarrow\left(n^{\prime}\right) \quad\left(n^{\prime}=6,7\right)$, the coefficients for $(n) \rightarrow\left(n^{\prime}\right)$ of $J a \operatorname{cobs}^{19}$ are used, neglecting the $l$ dependence in the case of $(n j) \rightarrow\left(n^{\prime}\right)$ for $n^{\prime}=6,7$. The coefficients for collisional de-excitation are computed from detailed balance.

The collisional ionization coefficients are taken from Jacobs. ${ }^{19}$ The $l$ dependence is included for $n=2$, but the $I$ averaged value (the only one given in Ref. 19) is used for $n=3$.

The coefficients for intralevel $\left(n=n^{\prime}\right)$ transitions among sublevels for $n=2,3$ are taken from Shevelko er al. ${ }^{22}$ (It should be noted that the expression for $\lambda^{2}$ in Ref. 22 contains a misprint, which is corrected by Tallents. $\left.{ }^{23}\right)$ Due to the small transition energies for the intralevel transitions, the ions as well as the electrons are effective in causing transitions. The transition rate is thus calculated including the effects of both ion and electron collisions.

Tabulated Einstein coefficients are used for the radiative decay rates. ${ }^{24,25}$ Only optically allowed, interlevel transitions are included. The $\left(j, j^{\prime}\right)$ dependence is handled in the same manner as for the collisional coefficients, namely by mutiplying the $\left(n^{\prime} l^{\prime}\right) \rightarrow(n l)$ Einstein coefficients by the angular factor $Q_{\kappa}\left(l^{\prime} j^{\prime}, l^{\prime}\right)$ with $k=1$, where necessary summed over $(j)$ and/or averaged over $\left(l^{\prime} j^{\prime}\right)$.

The radiative recombination coefficient for the hydrogenic ions is computed using the expression ${ }^{26}$

$$
A_{\infty n}=5.1 \times 10^{-14} Z\left(\frac{\beta}{n^{2}}\right)^{3 / 2} \exp \left(\frac{\beta}{n^{2}}\right) E_{1}\left(\frac{\beta}{n^{2}}\right) G_{n f} \quad \mathrm{~cm}^{3} / \mathrm{s} \text {, }
$$

where $\beta=13.6 Z^{2} / k T_{e}$ and $E_{1}(x)$ is the exponential integral function

$$
E_{1}(x)=\int_{x}^{\infty}\left(\frac{e^{-x}}{x}\right) d x
$$

Three-body recombination is calculated from detailed balance, as the inverse to collisional ionization. Dielectronic recombination from the hydrogenic to the helumlike ions is calculated using the expression given by Sobelman et al., ${ }^{15}$ which is an empirical fit to the results of detailed calculations. This expression is valid in the low-density limit, and overestimates somewhat the dielectronic recombination coefficient at high densities, since it does not allow for the reionization due to electron collisions of the highly excited intermediate state. However, it is sufficient for the present purposes, as the numerical results show that for the conditions of interest dielectronic recombination has little effect on the results.

All Gaunt factors [i.e., $G_{n f}$ in Eq. (3) and Eq. (22), $G_{g}$ in Eq. (9), and $G_{f n}$ in Eq. (13)] are set to 1.0 in the calculations. This is a reasonable approximation, since the Gaunt factors do not usually differ greatly from 1.0.

For the gain calculations, the line profle is assumed to consist of two superimposed components, as discussed above, the first of which has a homogeneous width given by $\Delta v_{1}=\Delta v_{e}+\Delta v_{i}$, and the second of which has a homogeneous width $\Delta v_{2}=\Delta v_{\mathrm{e}}+\Delta v_{i}+\Delta v_{I}$. Both profiles are assumed to be Voigt, with Doppler width $\Delta v_{D}$. The relative area ratio of the two components is $2: 1$ for $L$ yman- $\alpha$, and $1: 2$ for Baimer $\alpha 3 D_{5 / 2}-2 P_{3 / 2}$. The gain coefficient is caiculated from the expression 


$$
G=\left(\lambda^{2} A / 8 \pi\right) P(0)\left[N_{u}-\left(g_{u} / g_{l}\right) N_{i}\right],
$$

where $P(0)$ is the value of the normalized composite pronie at line center.

\section{RESULTS}

In this section, results for typical plasma characteristics, inversions, and gain coefficients are presented. Most of the Balmer- $\alpha$ gain resuits are given for a fuorine piasma, and most of the Lyman-a results for either a lithium or beryllium plasma. These results may be scaled to other elements using the well-known $\mathbb{Z}$-scaling laws for hydrogenic ions ${ }^{27}$ (although the scaing is only valid if the He-like population is negligible and all subleveis are populated statistically). For example, the gain coefficient varies with $Z$ as

$$
G \propto Z^{p} \times f\left(\eta_{e}, \theta_{e}, \overline{P_{1}}, H\right),
$$

where $\eta_{e}=N_{e} / Z^{7}, \quad \theta_{e}=T_{e} / Z^{2}, \quad \overline{P_{1}}=P_{1} / \mathbb{Z}^{4}$, and $H=h v / Z^{2}$. The exponent $p$ is between 6 and 7.5 , depending on the dominant broadening mechanism.

It may be shown that the power balance temperature exhibits a similar $Z$ scaling. All of the terms in Eq. (16) have the same $Z$ dependence, when expressed in terms of the reduced parameters $\eta_{e}, \theta_{c}, \overline{P_{1}}$, and $H$, and thus the power balance temperature scales according to

$$
T_{\mathrm{pb}}=Z^{2} \times f\left(\eta_{e}, \overline{P_{1}}, H\right)
$$

The results shown were in all cases calculated for $a$ photon energy of $15 Z^{2} \mathrm{eV}$ (i.e., approximately $10 \%$ higher than the ionization energy). This value was chosen somewhat arbitrarily. Ideally, the photon energy should be as close as possible to the ionization limis, both to maximize the coupling efficiency and to minimize the electron temperature (since the energy $h v-13.6 Z^{2} \mathrm{eV}$ is given up to the electrons in each ionization event). Some possible pump/lasant schemes could be expected to have a mean pump energy between $13.6 Z^{2}$ and $15 Z^{2} \mathrm{eV}$. For example, if H-like fuorine

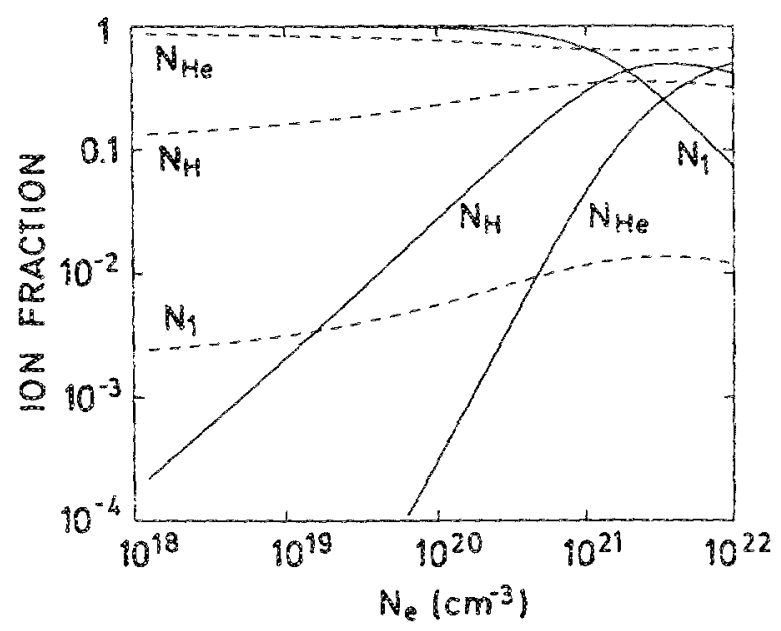

FIG. 1. Ion fractions for the bare nuclei $N_{i}$, H-ike ions $N_{H}$, and He-Hike ions $N_{\mathrm{HE}}$, without irradiation (dashed lines) and with irradiation (solid lines). $Z=9, T_{e}=150 \mathrm{eV}, I_{p}=5 \times 10^{1.3} \mathrm{~W} / \mathrm{cm}^{2}$, and $h v_{p}=1215 \mathrm{eV}$. were pumped with $2 \rightarrow 1$ radiation from He-like $\mathrm{Na}$, then $h v=1126.9$, or $13.91 Z^{2} \mathrm{eV}$. Such schemes would then have somewhat better gains for a given intensity than the results given here. Conversely, if the mean pump energy were significantly larger than $15 Z^{2} \mathrm{eV}$, then the results would be worse than those presented here.

In preserting the results, the electron density is taken to be a free parameter. In an actual situation, density gradients will exist in the plasma, in which case the inversion will be determined by the local value of the electron density. Also, the maximum achievable electron density will be determined by the critical density for the radiation used to create the plasma. For laser radiation at $0.65 \mu \mathrm{m}$, the critical density is $2.6 \times 10^{21} \mathrm{~cm}^{-3}$. If the plasma is created directly by $\mathrm{x}$-ray radiation, however, then all densities up to solid density are possible, since the critical density for $x$ rays is above solid density.

\section{A. Plasma characteristics}

Some typical characteristics of the steady-state plasmas produced by lonizing radiation are shown in Figs. 1-3. In Fig. 1 , the fractional ion populations of the bare nuclei, $H$ like ions, and He-like ions are shown for a fluorine plasma versus electron density with and without ionizing radiation. The temperature is held at $150 \mathrm{eV}$, and the pump intersity is $5 \times 10^{13} \mathrm{~W} / \mathrm{cm}^{2}$.

Without radiation, the plasma is dominated by the Helike ions. (The large He-like fraction indicates that lower ionization stages would also be present, if these had been included in the calculations.) In the presence of the ionizing radiation, however, the plasma is nearly fully stripped, with only a negligible He-like fraction for electron densities less than $10^{21} \mathrm{~cm}^{-3}$. These resuits illustrate the point that the plasma may have a significantiy higher ionization state in the presence of ionizing radiation than would normally be ex-

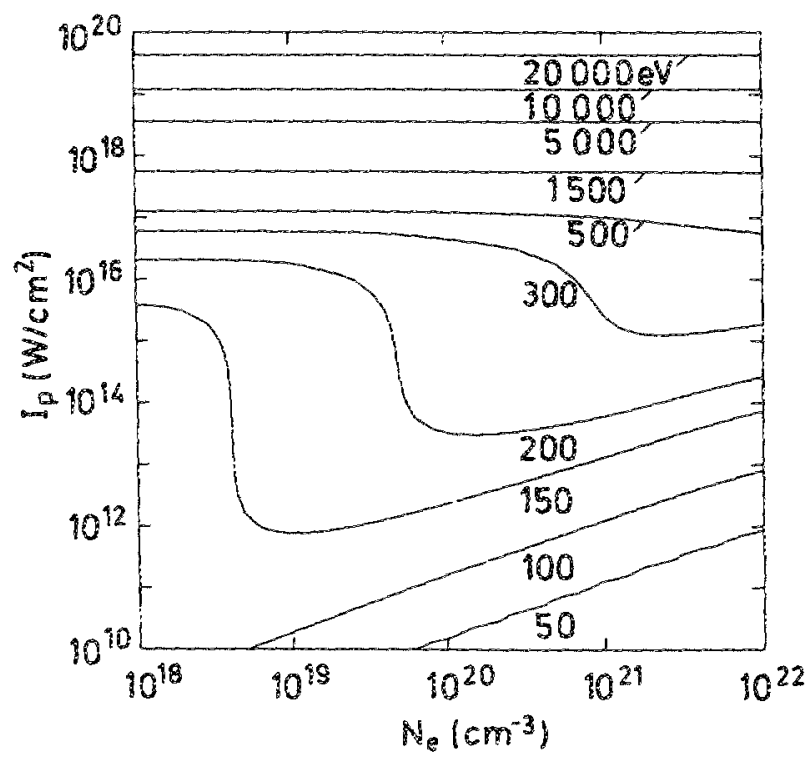

FIG. 2. Contours of power balance temperature ( $\mathrm{CV}), I_{p}$ is the pump intensity, and $N_{\theta}$ the electron density. $Z=9, h v_{p}=1215 \mathrm{eV}$. 


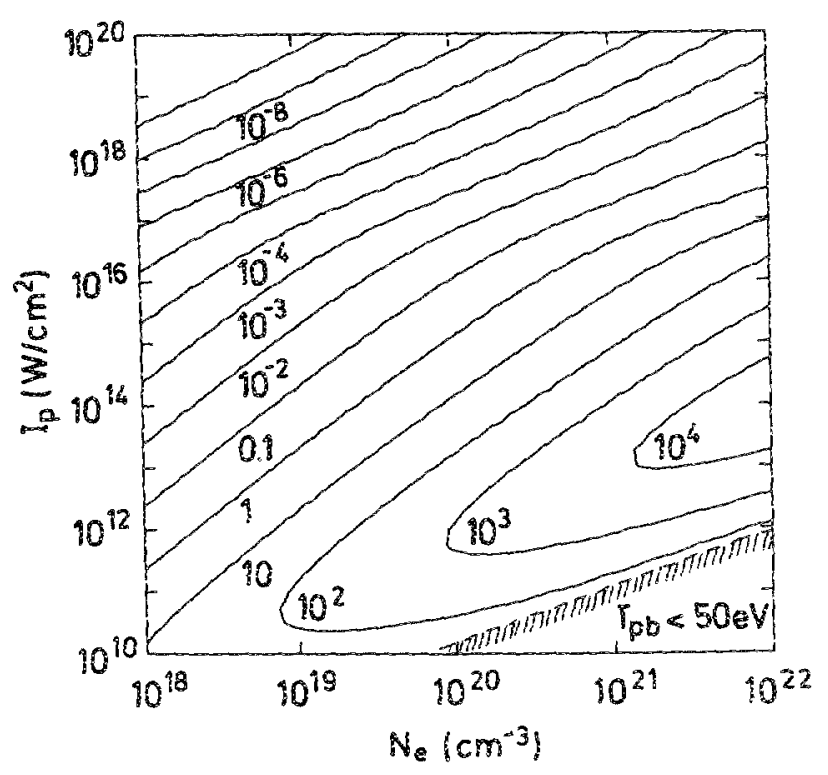

FIG. 3. Contours of Lyman- $\alpha$ line-eenter absorption coefficient $\left(\mathrm{cm}^{-1}\right)$ under power balance conditions. $Z=9, h v_{p}=1215 \mathrm{eV}$. (Results where $T_{\mathrm{pb}}<50 \mathrm{eV}$ not shown.)

pected for a given temperature, since the ionization baiance is maintained by the external pump radiation, rather than by electron collisions.

In Fig. 2, a contour plot of the power balance temperature $T_{\mathrm{pb}}$ is shown for a fuorine plasma versus electron density and pump intensity. For moderate intensities (say, less than $10^{18} \mathrm{~W} / \mathrm{cm}^{2}$ ), the temperature reached is in the neighborhood of $0.5-2.5 \times \mathcal{Z}^{2} \mathrm{eV}$. At very high intensities, the power balance temperature is independent of electron density. This results since the plasma is fully stripped, and the the only terms in Eq. (16) are $A_{f f}$ and $P_{f f}$, which both have the same dependence on $N_{e}$ and $N_{i}$.

A critical assumption in these calculations is that the plasma is optically thin. It is well known that optical trapping on the Lyman- $\alpha$ line can easily destroy Balmer- $\alpha$ inversions in hydrogenic ions. The effects of optical trapping may be accounted for in calculations by introducing escape probabilities, by which the Einstein coefficients are multiplied. For the escape probability for Lyman- $\alpha$ to be greater than $\approx 0.9$, the optical depth at line center for Lyman- $\alpha$ should be kept less than one. ${ }^{28}$

In Fig. 3, the values of the line-center absorption coeffcient $\kappa_{12}$ of the Lyman- $\alpha$ line are shown, in units of $\mathrm{cm}^{-1}$. These results indicate the maximum tolerable lateral dimension of the plasma for which optically thin results may be expected to apply. For example, on the contour where $\kappa_{12}$ $=10^{3} \mathrm{~cm}^{-1}$, the transverse plasma dimension must be held to less than $10 \mu \mathrm{m}$ for an optical depth less than one. It should be noted, however, that this does not take into account the bulk Doppler shifts due to strong velocity gradients within the plasma, which may relax the strict requirements on plasma size to some extent.

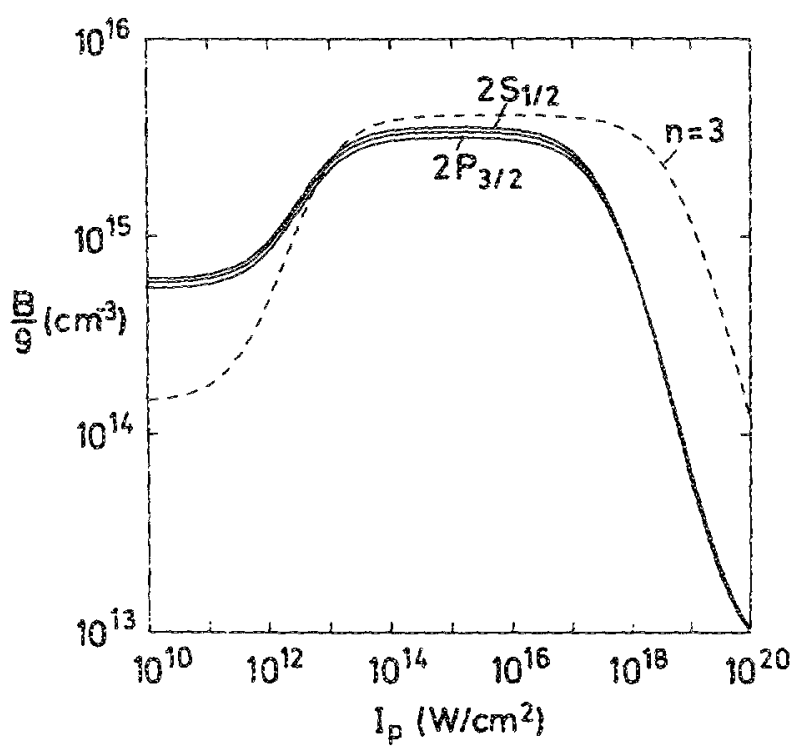

FIG. Af Efect of pump radiation on level populations. The quantity $B / g$ is the population density divided by the degeneracy. $Z=9, N_{c}=5 \times 10^{20}$ $\mathrm{cm}^{-3}, T_{e}=162 \mathrm{eV}$.

\section{Inversions}

The pumping effect of the ionizing radiation is illustrated in Figs. 4 and 5. In Fig. 4, the sublevel populations for $n=2$ and $n=3$ are shown (divided by their respective degeneracies) as a function of pump intensity for hydrogenic fuorine. The plasma temperature is held fixed at the value $162 \mathrm{eV}\left(=2 Z^{2} \mathrm{eV}\right)$ and the electron density is $5 \times 10^{20}$ $\mathrm{cm}^{-3}$. For $n=2$, all three sublevels are shown $\left(2 S_{1 / 2}, 2 P_{1 / 2}\right.$, and $2 P_{3 / 2}$ ), while for $n=3$ the sublevels are in statistical

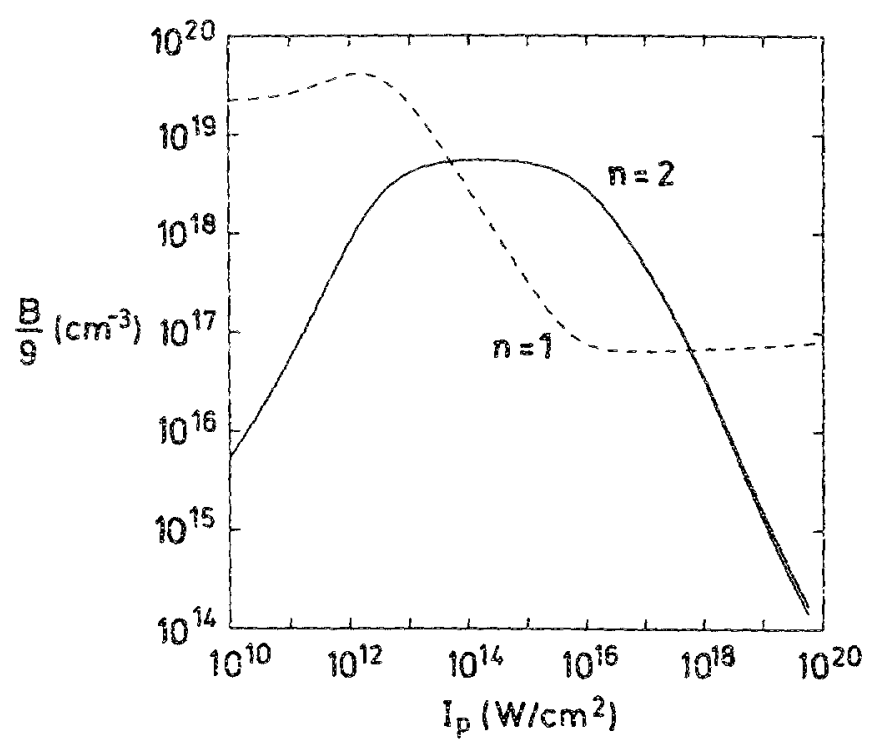

FIG. 5. Effect of pump radiation on level populations. $Z=3, N_{e}=5 \times 10^{20}$ $\mathrm{cm}^{-3}, T_{t}=9 \mathrm{eV}^{\mathrm{V}}$. 


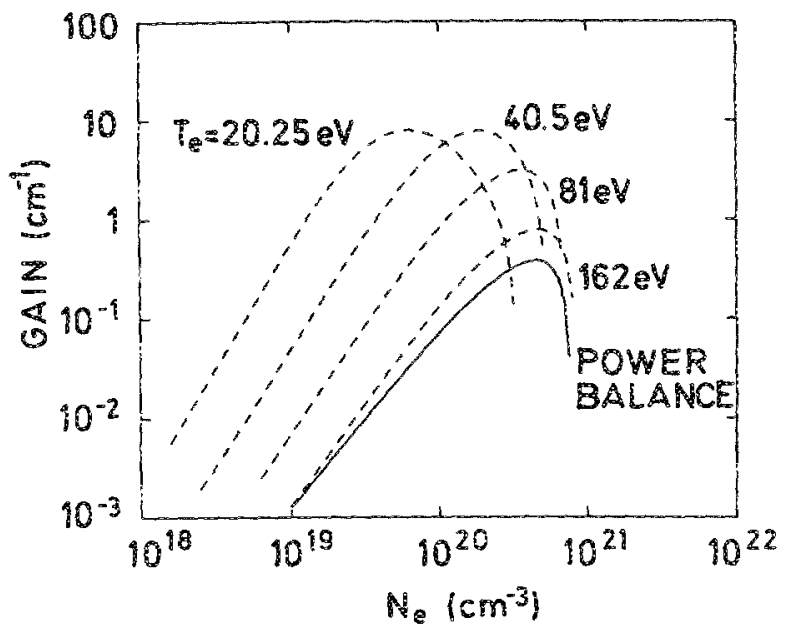

FIG. 6. Balmer- $\alpha$ gain vs electron density with the electron temperature as parameter. $Z=9$, pump intensity $5 \times 10^{13} \mathrm{~W} / \mathrm{cm}^{2}$. The temperatures are $0.25,0.5,1.0$, and $2.0 \times Z^{2}$. For the power balance case, the temperature varies along the curve within the range $2 Z^{2} e V-3 Z^{2} e V$.

equilibrium at this electron density, and thus have equal values of $B / g$.

At low intensities, the ionizing radiation has little effect, and the populations deviate only slightly from the values that they would have for $I_{p}=0$. At approximately $10^{12}$ $\mathrm{W} / \mathrm{cm}^{2}$, the populations of all levels begin to increase, as the He-like ions are stripped to hydrogenic ions. At an intensity of $10^{13} \mathrm{~W} / \mathrm{cm}^{2}$, the $3 D_{5 / 2}-2 P_{3 / 2}$ transition first becomes inverted. At much higher intensities $\left(>10^{17} \mathrm{~W} / \mathrm{cm}^{2}\right)$, the populations decrease, as direct photoionization out of the $n=2$ and $n=3$ states becomes strong.

The lack of statistical equilibrium among the $n=2 \mathrm{sub}$ states has an appreciable effect on the calculated inversion density, even for this relatively large electron density. For example, at $I_{p}=10^{14} \mathrm{~W} / \mathrm{cm}^{2}$, the inversion is twice as large for transitions ending on $2 P_{3 / 2}$ as it is for those ending on $2 S_{1 / 2}$.

In Fig. 5 , the level populations for $n=1$ and $n=2$ for hydrogenic lithium $(Z=3)$ are shown, again at an electron density of $5 \times 10^{20} \mathrm{~cm}^{-3}$, for a pump photon energy of 135 $\mathrm{eV}$. The electron temperature is held at $9 \mathrm{eV}(2-3$ times smaller than the power balance temperature). The threshold for positive inversion occurs at $5 \times 10^{12} \mathrm{~W} / \mathrm{cm}^{2}$, beyond which large fractional inversions may be obtained.

\section{Gain}

The Balmer- $\alpha 3 D_{5 / 2}-2 P_{3 / 2}$ gain coefficient for $Z=9$ is shown in Fig. 6 as a function of electron density for a pump intensity of $5 \times 10^{13} \mathrm{~W} / \mathrm{cm}^{2}$. The solid curve shows the gain obtained when the plasma temperature is determined by the power balance condition. The maximum gain for this case is approximately $0.4 \mathrm{~cm}^{-1}$. The dashed curves show the gain coefficient obtained if the plasma temperature is reduced by means of additional cooling to values below that given by the power balance condition. These curves show that decreasing the electron temperature results in a dramatic increase in the gain coefficient. At too low an electron temperature, how- ever, the pump is no longer strong enough to keep the plasma primarily in the hydrogenic ionization stage, and the gain again decreases.

The magnitude of the additional cooling obtainable duse to hydrodynamic expansion and/or conduction is estimated to be sufficient to hold $T_{e}$ to a value of $Z^{2} \mathrm{eV}$, which is less than half the power balance value (see Appendix). The primary requirement is to keep the transverse linear plasma dimension small-for the conditions assumed in the Appendix, less than approximately $50-70 \mu \mathrm{m}$. Since this constraint can be easily met, temperatures on the order of $Z^{2} \mathrm{eV}$ appear to be realistic values, and thus substantially higher gain than that predicted at the power balance temperature should be possible.

Figures 7 and 8 give an overview of the parameter regions where gain occurs on the Balmer-a transition. Contours of equal Baimer- $\alpha$ gain are shown for a fluorine plas$\mathrm{ma}$, where the abscissa and ordinate are the electron density and pump intensity, respectively. The gain coeficients shown in Fig. 7 were computed assuming the temperature to be given by the power balance condition. In Fig. 8 , the corresponding contour plot is shown for the case where the electron temperature is held at $81 \mathrm{eV}$.

In the regions below the dashed lines, more than $10 \%$ of the ions are in the He-like ionization stage. In these regions, the purnping radiation is not strong enough to hold the plasma in the hydrogenic ionization stage. It should be noted that the calculated results are only approximate where the He-like concentration is large, since lower ionization stages are not included.

The shapes of the contours in Figs. 7 and 8 may be easily understood physically. Increasing the pump power at fixed electron density, a threshold is first encountered, at which point the inversion first becomes positive. Raising the pump power beyond the threshold inereases the gain strongly, as the total hydrogenie population increases, due to increased

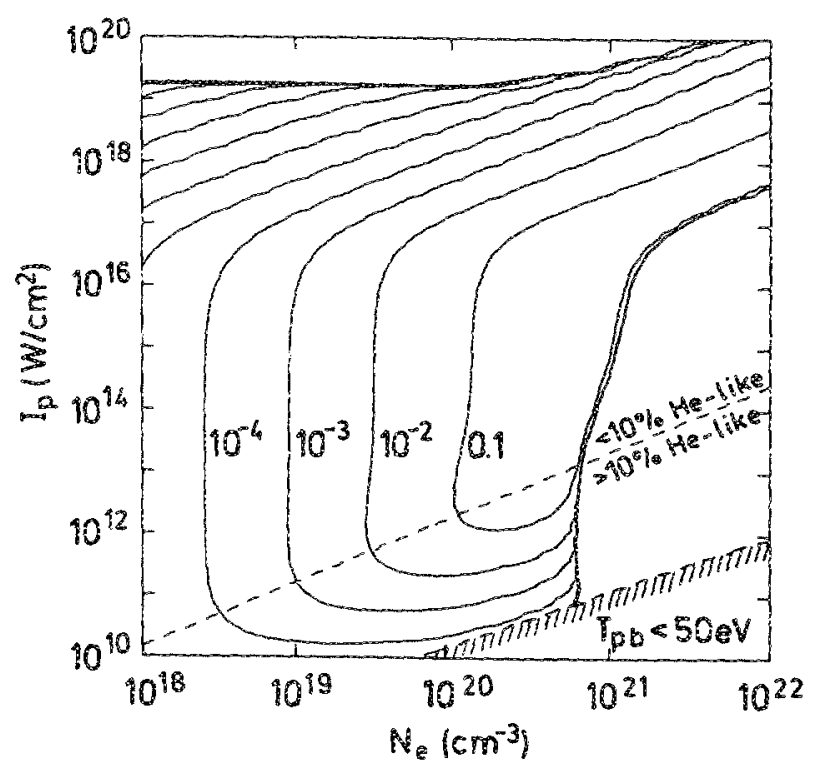

FIG. 7. Contours of equal Balmer- $\alpha$ gain under power balance conditions. $\mathcal{Z}=9, h v_{p}=1215 \mathrm{eV}$. (Results where $T_{p b}<50 \mathrm{eV}$ not shown.) 


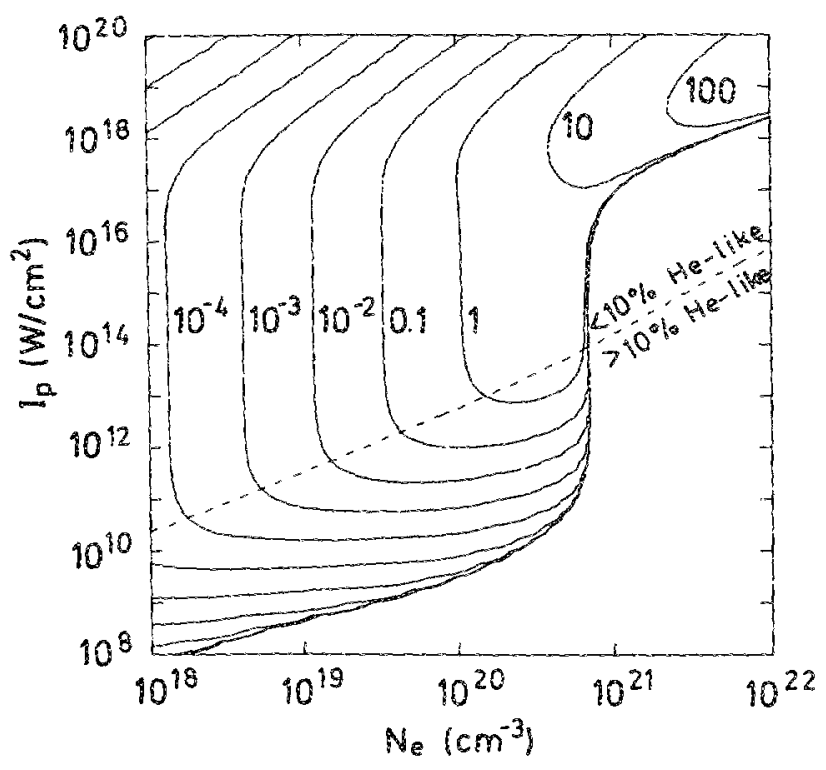

FIG. 8. Contours of equal Balmer- $\alpha$ gain with electron temperature fixed at $81 \mathrm{eV}, Z=9, h v_{p}=1215 \mathrm{eV}$. (Note that additional cooling is needed to achieve this temperature.)

ionization of the He-like ions. Once the He-like population has become negligible, the gain fattens out, and then decreases at very high pump rates, as the hydrogenic ions are stripped to bare nuclei. At still higher intensities (not shown in Fig. 8), the gain becomes negative again. This can be understood by recalling that at high pumping rates, the populations of the levels approach their saturation values and the level populations are not inverted [EG. (5)].

Keeping the pump power constant at a value in the range $10^{11}-10^{17} \mathrm{~W} / \mathrm{cm}^{2}$, the gain increases approximately quadratically with electron density, until a high electron density limit is reached $\left(\approx 10^{14} Z^{7} \mathrm{~cm}^{-3}\right)$, at which point the inversion is strongly quenched, as the collision-induced tran-

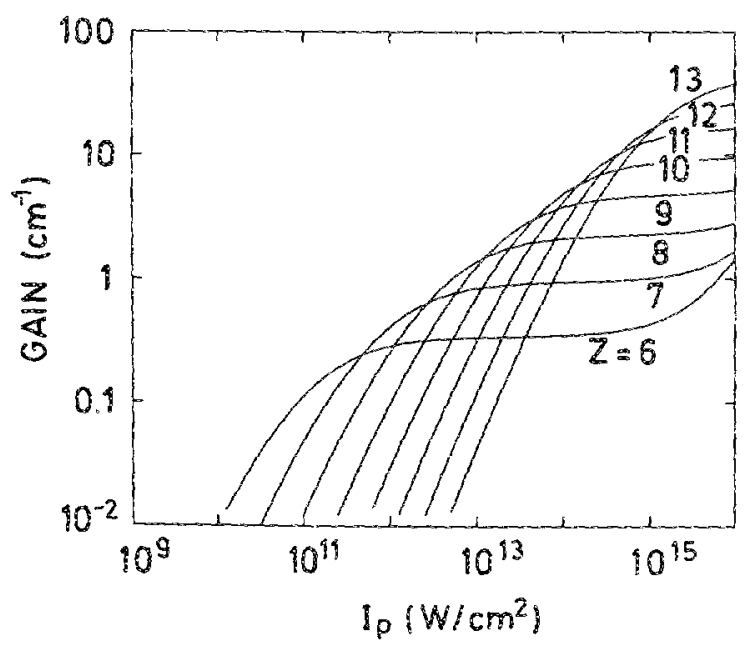

FIG. 9. Gain at optimum density vs pump intensily for various $Z(Z=6$ ro 13). Electron temperature $=Z^{2} \mathrm{eV}, h w_{p}=15 Z^{2} \mathrm{eV}$. (Note that additional cooling is needed to achieve this temperature.) sition rates begin to dominate over radiative decay, and the populations tend toward their Saha values. (However, at extremely high pump rates, this limit does not apply, since the lower level is depopulated directly by photoionization, rather than by radiative decay.)

The dependence of the peak gain on nuclear charge $Z$ is shown in Fig. 9, in which the gain coefficient at optimum electron density (where the gain is maximized; see Fig. 6) is shown versus pump power for $Z=6$ to $Z=13$. The temperature is taken to be $Z^{2} \mathrm{eV}$. $1 \mathrm{t}$ is seen that the gain increases considerably with $\mathcal{Z}$, but at the expense of a large rise in the threshold pump intensity. Using current high-power lasers, ar $x$-ray pump intensity of order $10^{13} \mathrm{~W} / \mathrm{cm}^{2}$ should be readily attainable in a line focus geometry (assuming a $1 \mathrm{~cm}$ length, a $20 \%$ conversion efficiency into $x$ rays, and a source plasma/target plasma separation of $\approx 200 \mu \mathrm{m})$. For this pump power level, $Z=8$ and $Z=9$ (with Balmer- $\alpha$ wavelengths of 103 and $81 \AA$, respectively) appear to offer the most promise.

Unlike the case for Balmer- $\alpha$, the Lyman- $\alpha$ transition does not invert if the plasma temperature is given by the power balance condition. Thus, additional cooling beyond radiative cooling alone is necessary for Lyman- $\alpha$ gain. However, if this can be achieved then large gain coefficients may be obtained, as seen in Fig. 10, in which contours of equal gain for the Lyman- $\alpha$ transition are shown for $Z=4$ and $k T_{e}=16 \mathrm{eV}$. The energy of the pump photons is $240 \mathrm{eV}$, and the wavelength of the laser is $75.9 \AA$. For the Lyman- $\alpha$ transition, there is no optimal electron density for the range shown here, as the gain continues to increase with increasing electron density.

Due to the high gain coefficients of the Lyman- $\alpha$ laser, a shorter line focus maybe used to obtain an acceptable gainlength product, with the double advantage that a higher $\mathrm{x}$ ray pump intensity can be generated with a given pump laser

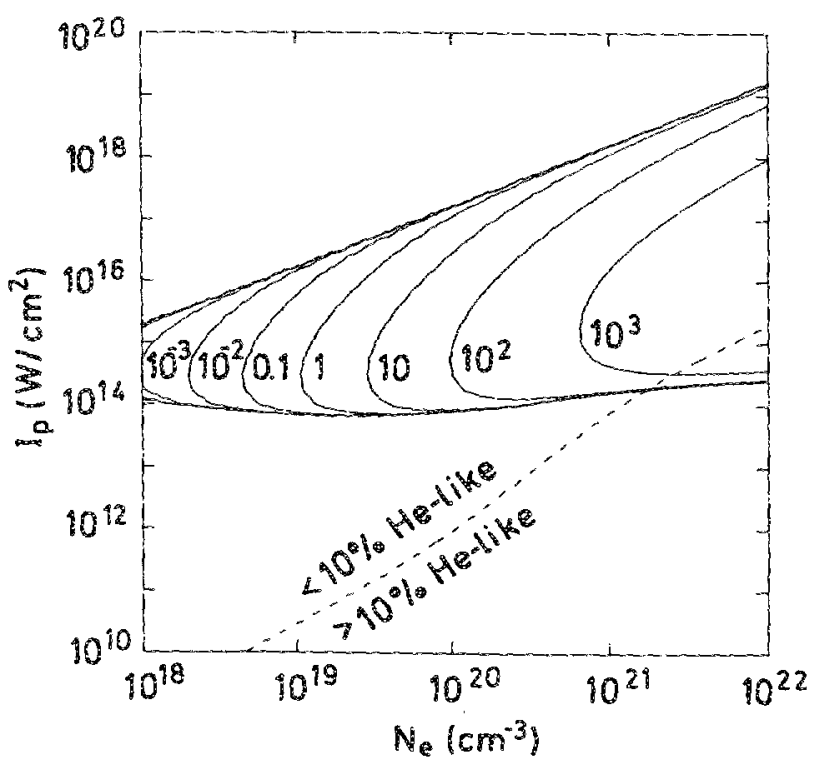

FIG. 10. Contours of equal Lyman- $\alpha$ gain. Electron temperature $16 \mathrm{eV}$, $Z=4$, h $v_{p}=240 \mathrm{eV}$. (Note that additional cooling is needed to achieve this temperature.) 
and beam refraction from electron density gradients is less problematic.

The Lyman- $\alpha$ gain coeficient is shown in Fig. 11 for $Z$ values from 3 to 6 plotted against pump power at an electron density of $5 \times 10^{20} \mathrm{~cm}^{-3}$. The plasma temperature is $Z^{2} \mathrm{eV}$. These results show that the primary difficulty in achieving lyman-a gain is in supplying enough pump power to overcome the high threshold values. By using a short line focus, however, the threshold values for $Z<5$ should be attainable.

\section{Y. CONCLUSIONS AMD OUTLOOK}

By means of numerical modeling, the conditions under which soft $\mathrm{x}$-ray gain occurs in a hydrogenic plasma subjected to ionizing electromagnetic radiation have been investigated. It is found that powers available from current high power lasers are sufficient to produce gain on hydrogenic Baimer- $\alpha$ lines for $\mathbb{Z}<10$, and on Lyman- $\alpha$ lines for $\mathbb{Z}<5$. In the case of the Balmer- $\alpha$ line, small but measurabie gain is predicted under conditions where the plasma temperature is determined solely by the radiative cooling of the plasma itself. If additional cooling is provided, the gain can be increased substantially. For the Lyman- $\alpha$ line, additional cooling of the electron gas is required to see any gain, but the gain coefficients become very large.

\section{ACKNOWLEDGMENTS}

This work was supported in part by the Commission of the European Communities in the framework of the Association Euratom/IPP.

\section{APPENDIX}

There are several ways to obtain a reduction of the electron temperature as required for higher gain, cooling by hy. drodynamic expansion and heat conduction to a nearby cold surface being the most promising ones. We note that radiative cooling by seeding the plasma with a high- $Z$ material ${ }^{29}$ will most likely not work in this case since the high- $Z$ ions

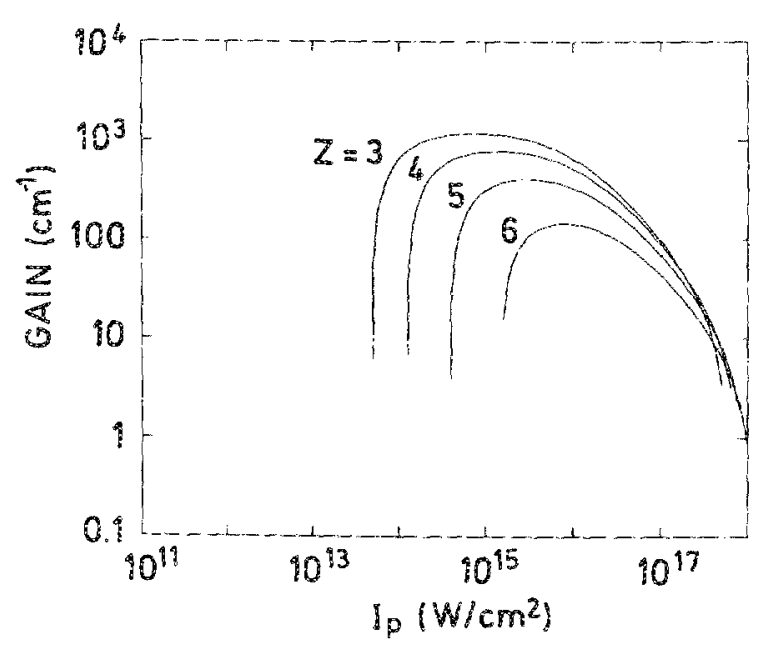

FIG. 11. Lyman- $\alpha$ gain vs pump intensity for $Z=3$ to $Z=6$. Electron temperature $=Z^{2}$ eV, electron density $=5 \times 10^{20} \mathrm{~cm}^{-3}, h x_{p}=15 Z^{2} \mathrm{eV}$. (Note that additional cooling is needed to achieve this temperature.) would also strongiy absorb the pump radiation and the net effect may be zero.

We estimate the cooling effect to be expected from hy drodynamic expansion and heat conduction. let the plasma cloud be a cylinder with radius $r$. The energy equation is ${ }^{30}$

$$
\frac{3}{2}\left(N_{e}+N_{i}\right) \pi r^{2} \frac{d k T}{d t}=-2 \pi r P r+\pi r^{2} \Delta W
$$

where the dot means differentiation with respect to time, $P$ is the plasma pressure, and $\triangle W$ is the difference between the absorbed and emitzed powers per $\mathrm{cm}^{3}$ (i.e., $\Delta W=A_{b f}+A_{f f}$ $\left.-P_{b b}-P_{b f}-P_{f f}\right)$. The quantity $\Delta W$ is strongly temperature dependent and becomes zero at the power balance temperature $T_{p b}$. By inserting for the pressure

$$
P=\left(N_{e}+N_{i}\right) k T
$$

one obtains

$$
\frac{d k T}{d t}=-\frac{4 k T r}{3 r}+\frac{2 \Delta W}{3\left(N_{e}+N_{i}\right)} .
$$

For expansion cooling to balance the heating by the pump radiation, we require that

$$
\frac{4 k T r}{3 r}=\frac{2 \Delta W}{3\left(N_{e}+N_{i}\right)} .
$$

With $Z=9, k T=81 \mathrm{eV}$, a pump intensity of $5 \times 10^{13}$ W/ $/ \mathrm{cm}^{2}$ and $N_{e}=3 \times 10^{20} \mathrm{~cm}^{-3}$ (the density for maximum gain from Fig. 6), the computer code gives

$$
\Delta W=7.6 \times 10^{12} \mathrm{~W} / \mathrm{cm}^{3} \text {. }
$$

Therefore from Eq. (A4)

$$
r / r=1.1 \times 10^{-9} \mathrm{~s} \text {. }
$$

If we estimate the expansion velocity as equal to the ion thermal velocity at this temperature $\left(3.3 \times 10^{6} \mathrm{~cm} / \mathrm{s}\right)$ then $r=36 \mu \mathrm{m}$, which is a physically realistic value. As long as the cylinder radius is kept at or below this value, hydrodynamic cooling is sufficient to of set radiative heating and hold the plasma temperature to $81 \mathrm{eV}$ or less.

To estimate the cooling by heat conduction, we neglect hydrodynamics and assume that only a small portion of the plasma is illuminated by the pump radiation, whereas the outer part is in contact with a cold material. We consider a cylindrical geometry, in which a small cylinder of radius $r_{0}$ is pumped, surrounded by a concentric cold cylinder of radius $R$. The heat flux is

$$
Q=-\kappa \frac{d T}{d r}
$$

with the themal conductivity given by ${ }^{12}$

$$
\kappa=4.67 \times 10^{-12} \frac{T^{5 / 2} \delta_{r}}{Z \ln \Lambda} \mathrm{cal} \mathrm{K}^{-1} \mathrm{~cm}^{-1} \mathrm{~s}^{-1} .
$$

In this equation in $\Lambda$ is the Coulomb logarithm and $\delta_{T}$ accounts for the non-Lorentzian nature of the gas. Taking $\ln \Lambda=5, Z=9$, and $\delta_{T}=0.7$ yields

$$
\kappa=\kappa_{0} T^{5 / 2},
$$

with

$$
\kappa_{0}=3 \times 10^{-13} \unlhd \mathrm{K}^{7 / 2} \mathrm{~cm}^{-1} \mathrm{~s}^{-1} .
$$


If the situation is stationary, the total heat flowing outward per second is constant at every radius, and thus

$$
2 \pi r Q(r)=\pi F_{0}^{2} \Delta W=\text { const. }
$$

From Eqs. (A7), (A8), and (A11) with the boundary condition $T(R)=0$, one obtains for the temperature

$$
T=T_{0}\left[\ln (r / R) / \ln \left(r_{0} / R\right)\right]^{2 / 7} \text {, }
$$

where $T_{0}$ is the temperature at $r=r_{0}$. The power transported by heat conduction becomes

$$
\pi r_{0}^{2} \Delta W=\frac{4 \pi \kappa_{0} T_{0}^{\tau / 2}}{7 \ln \left(R / r_{0}\right)} \mathrm{W} / \mathrm{cm}
$$

Taking $R / r_{0}=10$ and again $k T_{0}=81 \mathrm{eV}$, the power conducted to the outer cylinder per cm of axial length is

$$
P=\pi r_{0}^{2} \Delta W=1.9 \times 10^{8} \mathrm{~W} / \mathrm{cm} .
$$

With $\Delta W=7.6 \times 10^{12} \mathrm{~W} / \mathrm{cm}^{3}$ at $81 \mathrm{eV}$, one concludes that an irradiated inner cylinder with a maximum radius of $r_{0}$ $=28 \mu \mathrm{m}$ can be cooled to $81 \mathrm{eV}$ by heat conduction.

'D. L. Matthews, P. L. Hagelstein, M. D. Rosen, M. I. Eckart, N. M. Ceglio, A. U. Hazi, H. Medecki, B. J. MacGowan, J. E. Trebes, B. L. Whitten, E. M. Campbell, C. W. Hatcher, A. M. Hawryluk, R. L. Kaufman, L. D. Pleasance, G. Rambach, I. Scofield, G. Stone, and T. A. Weaver, Phys. Rev. Lett. 54, 110 (1985).

${ }^{2}$ B. J. MacGowan, M. D. Rosen, M. J. Eckari, P. I. Hagelstein, D. L. Matthews, D. G. Nilson, T. W. Phillips, J. H. Scofield, G. Shimkaveg, J. E. Trebes, R. S. Walling, B. L. Whitten, and J. G. Woodworth, J. Appl. Phys. 61, $5243(1987)$.

3. J. MacGowan, S. Maxon, P. L. Hagelstein, C. J. Keane, R. A. London, D. L. Matthews, M. D. Rosen, I. H. Scofield, and D. A. Whelan, Phys. Rev, Lett. 59, 2157 (1987).

${ }^{4}$ S. Suckewer, C. H. Skinner, H. Milchberg, C. Keane, and D. Vorhees, Phys. Rev. Lett. 55, 1753 (1985).
${ }^{5}$ M. H. Key, J. E. Boon, C. Brown, C. Chenai-Popovics, R. Corbeti, A. R. Damerell, P. Gottfeldt, C. J. Hooker, G. P. Kiehn, C. L. S. Lewis, D. A. Pepler, G. J. Pert, C. Reagan, S. J. Rose, I. N. Ross, P. T. Rumsby, S. Sadaat, R. Smith, T. Tomi, and O. Willi, I. Phys. (Paris) Colloq. 6, 71 (1986).

${ }^{6}$ P. L. Hagelstein, Plasma Phys. 25, 1345 (1983).

${ }^{7}$ R. H. Dixon and R. C. Elton, J. Opt. Soc. Am. B 1, 232 (1984).

${ }^{8}$ F. V. Bunkin, V. I. Derzhiev, and S. I. Yakovienko, Sov. J. Quantum Electron. 1, 971 (1981).

'V. A. Boiko, F. V. Bunkin, V. I. Derzhiev, and S. I. Yakovlenko, IEEE J. Quantum Electron. QE-20, 206 (1984).

10E. E. Fill, J. Phys. (Paris) Collog. 6, 117 (1986)

${ }^{1} \mathrm{G}$. J. Pert, Plasma Phys. Contr. Fusion 27, 1427 (1985).

${ }^{12} \mathrm{~L}$. Spitzer, Physics of Fully Ionized Gases (Interscience, New York, 1956).

${ }^{13}$ D. Mihalas, Stellat Atmospheres (Freeman, San Francisco, 1978), p. 95.

${ }^{14}$ D. H. Sampson, J. Phys. B 10, 749 (1977).

151. I. Sobelman, L. A. Vainshtein, and E. A. Yukov, Excitation of Atoms and Broadening of Spectral Lines (Springer, Berlin, 1981).

${ }^{16}$ H. R. Griem, Spectrai Line Broadening by Plasmas (Academic, New York, 1974\}, p. 8.

${ }^{17}$ R. Stamm, B. Talin, E. L. Pollack, and C. A. Iglesias, Phys. Rev. A 34, 4144 (1586).

${ }^{18}$ D. H. Oza, R. L. Greene, and D. E. Kelleher, Phys. Rev. A 34, 4519 (1986).

${ }^{19}$ A. Jacobs, J. Quant. Spectrosc. Radiat. Transfer 12, 243 (1972).

${ }^{20}$ L. B. Golden, R. E. H. Clark, S. I. Goett, and D. H. Sampson, Astrophys. J. Suppi. Series 45, 603 (1981).

${ }^{21}$ R. E. H. Clark, D. H. Sampson, and S. J. Goett, Astrophys. J. Suppl. Series 49, 545 (1982).

${ }^{22}$ V. P. Shevelko, I. Yu. Skobelev, and A. V. Vinogradov, Phys. Scr. 16, 123 (1977).

23. G. J. Tallents, J. Phys. (Paris) Colloq. 6, 151 (1986).

${ }^{24}$ W. L. Wiese, M. W. Srnith, and B. M. Glennon, Atomic Transition Probabilities, Vol. 1 (National Bureau of Standards, Washington, DC, 1966).

${ }^{25}$ L. C. Green, P. P. Rush, and C. D. Chandler, Astrophys. J. Suppl. 3, 37 (1957).

${ }^{26}$ M. J. Seaton, Mon. Not. Astron. Soc. 119, 81 (1959).

${ }^{27}$ R. W. P. McWhirter and A. G. Hearn, Proc. Phys. Soc. 82, 641 (1963).

${ }^{38}$ H. W. Drawin and F. Emard, Beitr. Plasma Phys. 13, 143 (1973).

${ }^{29}$ J. P. Apruzese, J. Davis, P. C. Kepple, and M. Blaha, J. Phys. (Paris) Colloq. 6, 15 (1986).

${ }^{30}$ K. M. Dawson, Phys. Fluids 7, 981 (1964). 